\title{
EDITORIAL
}

\section{Knotting the NETs: Analyzing histone modifications in neutrophil extracellular traps}

\author{
Indira Neeli and Marko Radic* \\ See related research by Liu et al., http://arthritis-research.com/content/14/1/R25
}

\begin{abstract}
Neutrophil extracellular chromatin traps (NETs) are a recently described mechanism of innate immune responses to bacteria and fungi. Evidence indicates that NETs are induced by inflammation, that they contribute to diverse disease pathologies, and that they associate with bactericidal substances. Genomic DNA is released in NETs, leading to a cell death that has been labeled NETosis. Although NETosis clearly differs from apoptosis, the classical form of cell death, recent experiments indicate a connection between NETosis and autophagy. The regulated deployment of NETs may require covalent modification of histones, the basic DNA-binding proteins that organize chromatin in the cell's nucleus and within NETs. Histone modification by peptidylarginine deiminase 4 (PAD4) is necessary for NET release. The functions of additional histone modifications, however, remain to be tested.
\end{abstract}

Less than a decade since their discovery, neutrophil extracellular traps (NETs) remain in the headlines. Initially, interest focused on the structure of extracellular NET chromatin and its capacity to capture and damage bacteria. Soon, however, researchers began to see the implications of extracellular chromatin for the development of autoimmune diseases. One quintessential autoimmune disease, systemic lupus erythematosus (SLE), is known to arise together with autoantibodies to DNA and chromatin, although the immediate trigger for the production of these autoantibodies is unclear. A connection between NETs and autoimmunity was made by discovering that histones, a set of proteins that act as a structural harness for DNA in chromatin, are modified

*Correspondence: mradic@uthsc.edu

Department of Microbiology, Immunology and Biochemistry, University of Tennessee Health Science Center, 858 Madison Avenue, Memphis, TN 38163, USA by peptidylarginine deiminase 4 (PAD4), an enzyme that converts arginines to citrullines. Researchers had long suspected that autoantigen modifications could provide the initial stimuli in autoimmunity because subtle alterations in a protein's primary sequence can break tolerance. PAD4 is implicated in the development of rheumatoid arthritis (RA) because the most reliable clinical test for RA uses the detection of anti-citrulline antibodies in the sera of patients.

In a sophisticated set of experiments reported in the previous issue of Arthritis Research \& Therapy, Liu and colleagues [1] accomplished an extensive inventory of post-translational modifications in NET histones. The researchers induced NETs from human neutrophils, as well as two cell lines that assume neutrophil-like characteristics, and used a panel of 40 commercially available antisera to identify histone modifications that arise in parallel with NETs. Stimuli that were used to elicit NET release also induced histone $\mathrm{H} 3$ and $\mathrm{H} 4$ citrullination in human neutrophils and the EPRO cell line. However, other modifications such as histone H4 lysine 20 methylation and $\mathrm{H} 4$ lysine 16 acetylation showed inconsistent results in neutrophils versus the EPRO cells. To survey histone modifications, Liu and colleagues [1] confronted technical difficulties in that histone amino terminal tails contain the highest concentration of histone modifications yet are also highly susceptible to proteases secreted by activated neutrophils [2,3]. The histone tails act as flexible tethers that organize chromatin into higher-order structures. Interestingly, purified NETs failed to induce an immune response in mice, although a subset of SLE sera reacted strongly with citrullinated histone $\mathrm{H} 3$ [1]. Therefore, mechanisms that regulate histone modification deserve further attention.

Neeli and colleagues [4] were the first to identify citrullinated histone H3 in NETs, a discovery that was confirmed by others [5]. Neeli and colleagues [4] provided a second important insight, namely that PAD4citrullinated histone $\mathrm{H} 3$ is a reliable marker of inflammation. Thus, it became clear that the release of NETs is not an 'accident' caused by a barrage of proteases and 
reactive oxygen species unleashed from neutrophils. Instead, production of NETs requires enzymatic activity and input from neutrophil surface receptors and the cytoskeleton [6]. By analyzing PAD4-deficient mice, Li and colleagues [7] demonstrated that PAD4 is essential for the production of NETs in response to bacterial infections. The regulation of PAD4 activity thus moved to the forefront of the research on NETs.

It is now clear that NET release takes advantage of NADPH (nicotinamide adenine dinucleotide phosphate) oxidase and the main granule proteases to trigger and construct the extended chromatin network $[3,8]$. In addition, myeloperoxidase is found in NETs after their release from the cells, and this enzyme and its products are the main components in NETs that kill bacteria [9]. In a notable study from the labs of Banchereau and Pascual [10], it was reported that SLE neutrophils are poised to undergo NETosis upon stimulation with anti-ribonucleoprotein autoantibodies and that NETs released by these neutrophils contain LL37 and HMGB-1, well-known stimulators of immune responses. In subsequent analyses using sera from patients with connective tissue disease, anti-citrullinated histone antibodies were observed in Felty's syndrome, a rare disorder that shares serologic features with RA and SLE, whereas such autoantibodies were infrequent in SLE and RA [11]. These findings indicate that the process of NETosis is highly relevant to the development of human autoimmune responses, although a direct cause and effect may not connect the release of NETs to the production of autoantibodies.

The detailed characterization of NET histone modifications, as accomplished by Liu and colleagues [1], invites speculations about the possible functions of these modifications. Several questions deserve further study: Will NET histone modifications, such as methylation, acetylation, and citrullination, be found to participate in gene regulation that sets the stage for NET release? Will the primary function of histone modifications turn out to be the decondensation of nuclear chromatin that is required for NETs expand to their optimal size and internal structure? Alternatively, NET histone modifications may serve non-traditional purposes. For example, certain modifications may anchor other NET components such as elastase, LL37, or myeloperoxidase to the chromatin meshwork. Unique modifications in NETs may attract phagocytes and stimulate them to ingest the trapped microorganisms. Other histone modifications may activate or dampen the inflammatory response by acting on innate pattern recognition receptors. The answers to these questions will, no doubt, keep research on NETs in leading immunology and microbiology journals for years to come.

\section{Abbreviations}

NET, neutrophil extracellular trap; PAD4, peptidylarginine deiminase 4; RA rheumatoid arthritis; SLE, systemic lupus erythematosus.

\section{Competing interests}

The authors declare that they have no competing interests.

\section{Authors' contributions}

Both authors contributed to the writing and editing of the text.

\section{Acknowledgments}

The authors acknowledge the research support from the Lupus Research Institute of New York and the Dana Foundation for Human Immunology. We apologize for our highly focused and therefore incomplete survey of research accomplishments in NET research.

Published: 6 April 2012

\section{References}

1. Liu CL, Tangsombatvisit S, Rosenberg JM, Mandelbaum G, Gillespie EC, Gozani OP, Alizadeh AA, Utz PJ: Specific post-translational histone modifications of neutrophil extracellular traps as immunogens and potential targets of lupus autoantibodies. Arthritis Res Ther 2012, 14:R25.

2. Urban CF, Ermert D, Schmid M, Abu-Abed U, Goosmann C, Nacken W, Brinkmann V, Jungblut PR, Zychlinsky A: Neutrophil extracellular traps contain calprotectin, a cytosolic protein complex involved in host defense against Candida albicans. PLoS Pathog 2009, 5:e1000639.

3. Papayannopoulos V, Metzler KD, Hakkim A, Zychlinsky A: Neutrophil elastase and myeloperoxidase regulate the formation of neutrophil extracellular traps. J Cell Biol 2010, 191:677-691

4. Neeli I, Khan SN, Radic M: Histone deimination as a response to inflammatory stimuli in neutrophils. J Immunol 2008, 180:1895-1902.

5. Wang Y, Li M, Stadler S, Correll S, Li P, Wang D, Hayama R, Leonelli L, Han H, Grigoryev SA, Allis CD, Coonrod SA: Histone hypercitrullination mediates chromatin decondensation and neutrophil extracellular trap formation. J Cell Biol 2009, 184:205-213.

6. Neeli I, Dwivedi N, Khan S, Radic M: Regulation of extracellular chromatin release from neutrophils. J Innate Immun 2009, 1:194-201.

7. Li P, Li M, Lindberg MR, Kennett MJ, Xiong N, Wang Y: PAD4 is essential for antibacterial innate immunity mediated by neutrophil extracellular traps. J Exp Med 2010, 207:1853-1862.

8. Vethanayagam RR, Almyroudis NG, Grimm MJ, Lewandowski DC, Pham CT, Blackwell TS, Petraitiene R, Petraitis V, Walsh TJ, Urban CF, Segal BH: Role of $\mathrm{NADPH}$ oxidase versus neutrophil proteases in antimicrobial host defense. PloS One 2011, 6:e28149.

9. Parker H, Albrett AM, Kettle AJ, Winterbourn CC: Myeloperoxidase associated with neutrophil extracellular traps is active and mediates bacterial killing in the presence of hydrogen peroxide. J Leukoc Biol 2012, 91:369-376.

10. Garcia-Romo GS, Caielli S, Vega B, Connolly J, Allantaz F, Xu Z, Punaro M, Baisch J, Guiducci C, Coffman RL, Barrat FJ, Banchereau J, Pascual V: Netting neutrophils are major inducers of type I IFN production in pediatric systemic lupus erythematosus. Sci Trans/ Med 2011, 3:73ra20.

11. Dwivedi N, Upadhyay J, Neeli I, Khan S, Pattanaik D, Myers L, Kirou KA, Hellmich B, Knuckley B, Thompson PR, Crow MK, Mikuls TR, Csernok E, Radic $M:$ Felty's syndrome autoantibodies bind to deiminated histones and neutrophil extracellular traps. Arthritis Rheum 2011 Oct 27 [Epub ahead of print].

doi:10.1186/ar3773

Cite this article as: Neeli I, Radic M: Knotting the NETs: Analyzing histone modifications in neutrophil extracellular traps. Arthritis Research \& Therapy 2012, 14:115 